\title{
Una estimación del costo y cambios en el bienestar de los colombianos con el nuevo Plan de Beneficios en Salud*
}

\author{
Recibido: 21 de octubre de 2013 - Aceptado: 16 de octubre de 2014
}

Doi: dx.doi.org/10.12804/rev.econ.rosario.17.02.2014.04

\author{
Álvaro J. Riascos ${ }^{\dagger}$ \\ Sergio A. Camelo $\ddagger$ \\ Universidad de los Andes y Quantil SAS
}

Resumen

En este artículo se estima el costo esperado del nuevo Plan de Beneficios en Salud. El análisis parte de la base de que al disminuir las barreras para la reclamación de servicios que en la actualidad se consideran por fuera del Plan Obligatorio de Salud (POS) -denominados aquí No POS- habrá una recomposición de la demanda por servicios del POS y del No POS. La aproximación metodológica utilizada consiste en la estimación de un modelo de elección discreta

* Agradecemos el apoyo financiero e información suministrada por el Ministerio de Salud para la realización de este trabajo. Igualmente, agradecemos los comentarios del Ministerio de Hacienda, Departamento de Planeación Nacional, Luis Gonzalo Morales, Ramón Abel Castaño, Alejandro Gaviria y los asistentes al Seminario CEDE, de la Universidad de los Andes. Una versión preliminar (presentación) de este trabajo circuló con el título "Un modelo de elección discreta para simular el costo del Plan Integral en Salud". Todos los errores y opiniones son responsabilidad exclusiva de los autores y no comprometen al Ministerio de Salud.

+ Facultad de Economía. Correo electrónico: ariascos@uniandes.edu.co. Dirección: Facultad de Economía, Universidad de los Andes. Carrera 1A \# 18A-70, Bloque C, Bogotá D. C.

‡ Facultad de Economía y Departamento de Matemáticas. Correo electrónico: sa.camelo38@uniandes.edu.co. Dirección: Facultad de Economía, Universidad de los Andes. Carrera 1A \# 18A-70, Bloque C, Bogotá D. C.

Para citar este artículo: Riascos, A. J., \& Camelo, S. A. (2014). Una estimación del costo y cambios en el bienestar de los colombianos con el nuevo Plan de Beneficios en Salud. Revista de Economía del Rosario, 17(2), 229-314. doi: dx.doi.org/10.12804/rev.econ.rosario.17.02.2014.04 
que, con base en las elecciones observadas de reclamaciones y consumo de servicios POS y No POS en el ámbito individual, permite estimar las preferencias de los individuos. Este modelo permitirá, además, hacer un análisis de la variación en el bienestar de los individuos antes y después de la reforma. Puesto que el modelo se estima por edad, género y diagnosis (dentro de un grupo de 29 enfermedades crónicas), es posible estimar los cambios en bienestar en distintos grupos de riesgo. Los resultados muestran que el aumento esperado en el costo del nuevo plan de beneficios será a lo sumo del 16\%, con respecto al costo actual (POS más No POS del régimen contributivo), con una desviación estándar de 7,10\%, entre los grupos de riesgo. En 2010 el costo del POS más No POS del régimen contributivo fue aproximadamente de 12 billones de pesos, con lo que el costo del nuevo plan de beneficios sería a lo sumo 2 billones de pesos, con una desviación estándar de 0,9 billones. Se calcula que el excedente del consumidor aumentará en promedio 5,73 veces, lo que aumentará el gasto y sugiere que el nuevo plan es muy eficaz en generar bienestar por unidad de gasto. Los resultados también identifican los grupos de riesgo que más costarán, que más aumentarán su bienestar y para los cuales el aumento en bienestar por unidad de gasto adicional es más alto. Este trabajo es una aproximación rigurosa a un problema complejo de construcción de un escenario contra factual (posreforma). A diferencia de un modelo de elección discreta estándar, el análisis realizado presenta complicaciones, pues no es posible observar las elecciones de todos los individuos frente a las diferentes alternativas (por ejemplo, no se observó la escogencia de servicios No POS de individuos que, bajo el actual sistema, han elegido únicamente servicios POS). El problema se resuelve mediante el ajuste de un modelo econométrico capaz de estimar las elecciones no observadas de los individuos.

Clasificación JEL: I11, I13, I18

Palabras clave: reforma salud Colombia, nuevo plan de beneficios, modelos de elección discreta.

\title{
An Estimation of the Costs and Welfare Benefits of the New Colombian Health Insurance Plan
}

\begin{abstract}
This article estimates the expected cost and welfare of the new benefits plan in Colombian public health care plan. The analysis is based on the idea that by reducing barriers to claims for services that are currently outside of the Mandatory Health Plan (POS, Plan Obligatorio de Salud), here called non-POS, there will be recomposition of the demand of POS and non-POS health services. The methodological approach used of the estimation is a discrete choice model that, based on the observed choices of claims and consumption of POS and non-POS services at the individual level, enables an estimation of the revealed preferences of individuals and a prediction of the new demand after the health reform. Since the model is estimated for each risk group (age, sex, and chronic diseases), it is possible to estimate the changes in cost and welfare by risk group. The results show that on average the expected increase in the cost of the new benefits plan is $16 \%$ compared to the current cost (POS plus non-POS), with a standard deviation of $7,10 \%$ among risk groups. However, consumer surplus is on average 5,73 times greater than the increase in spending. This suggests that the new plan is very effective in
\end{abstract}


generating consumer surplus per additional spending unit. The result also identifies the risk groups that will cost the most, and for which the increase in well-being per additional spending unit will be the highest. Unlike a standard discrete choice model, the analysis conducted presents additional complications, as it is not possible to observe the utility of individuals for all choice alternatives (e.g., spending on non-POS services for individuals who choose only POS services is not observed). It is thus necessary to estimate these values.

JEL Classification: I11, I13, I18

Keywords: colombian health reform, new benefits plan, discrete choice models.

\section{Uma estimação do custo e mudanças em bem-estar dos colombianos com o novo plano de benefícios em saúde}

\section{Resumo}

Neste artigo estima-se o custo esperado do novo plano de benefícios em saúde. A análise parte da base de que ao diminuir as barreiras para a reclamação de serviços que na atualidade consideram-se por fora do Plano Obrigatório de Saúde (POS), denominados aqui Não POS, haverá uma recomposição da demanda por serviços do POS e do Não POS. A aproximação metodológica utilizada consiste na estimação de um modelo de eleição discreta que com base nas eleições observadas de reclamações e consumo de serviços POS e Não POS no nível individual, permite estimar as preferências dos indivíduos. Este modelo permitirá, além, fazer uma análise da variação no bem-estar dos indivíduos antes e depois d reforma. Devido a que o modelo estima-se por idade, gênero e diagnose (dentro de um grupo de 29 doenças crónicas), é possível estimar as mudanças em bem-estar em diferentes grupos de risco. Os resultados mostram que o aumento esperado no custo do novo plano de benefícios será no máximo 16\% com respeito ao custo atual (POS mais Não POS do regime contributivo), com um desvio padrão de 7,10\% entre os grupos de risco. No ano 2010 o custo do POS mais Não POS do regime contributivo foi aproximadamente 12 bilhões de pesos, com o que o custo do novo plano de benefícios seria no máximo 2 bilhões de pesos, com um desvio padrão de 0.9 bilhões. Calculasse que o excedente do consumidor aumentará em média 5,73 vezes o que aumentará o gasto, o que sugere que o novo plano é muito eficaz em gerar bem-estar por unidade de gasto. Os resultados também identificam os grupos de risco que mais custarão, mais aumentarão seu bem-estar e para os quais o aumento de bem-estar por unidade de gasto adicional é mais alto. Este trabalho é uma aproximação rigorosa a um problema complexo de construção de um cenário contra factual (pós-reforma). A diferença de um modelo de escolha discreta padrão, a análise realizada apresenta complicações pois não é possível observar as escolhas de todos os indivíduos frente às diferentes alternativas de (por exemplo, não observamos a escolha de serviços Não POS de indivíduos que sob o sistema atual têm escolhido unicamente serviços POS. O problema resolve-se mediante o ajuste de um modelo econométrico capaz de estimar as eleições não observadas dos indivíduos.

Classificação JEL: I11, I13, I18

Palavras-chave: reforma saúde colômbia, novo plano de benefícios, modelos de escolha discreta. 


\section{Introducción}

En Colombia, el Plan Obligatorio de Salud (POS) está definido por una lista positiva de servicios, procedimientos y medicamentos garantizados por la ley para todas las personas aseguradas por una Empresa Prestadora de Salud (EPS). Esta lista, sin embargo, tiene algunas limitaciones. Si un paciente requiere de un servicio no contemplado en el POS (a estos servicios los denominaremos No POS), entonces el paciente deberá prepararse para una larga serie de trámites donde existe la posibilidad de no obtener el servicio o medicamento. La vía más común por la que se obtienen servicios No POS es por una tutela al Estado, que, de ser exitosa, garantiza el pago de todos los servicios al usar fondos del Estado. Como son las EPS las que proveen los servicios, estas cobran el dinero de los servicios No POS al Estado. A estos cobros se les llamarán recobros.

El sistema actual presenta serios problemas. Primero, la decisión respecto a si se otorga o no un servicio No POS por medio de una tutela es tomada por un juez, quien puede estar informado de las condiciones médicas del paciente, pero que nunca tendrá el criterio de un médico. Segundo, los servicios No POS son proveídos por el Estado, así que no existe competencia en precios y servicios, como sí existe en medicamentos POS. La inexistencia de competencia tiene un fuerte efecto sobre las finanzas del Estado, al considerar que los recobros son una porción importante del gasto en salud. Tercero, los pesados trámites administrativos en la obtención de servicios No POS pueden desincentivar a pacientes de la reclamación de servicios necesarios para su salud.

Debido a estos problemas, se ha propuesto unificar el acceso a servicios del POS y del No POS al crear una lista mucho más amplia de servicios garantizados por la ley y que deberán ser responsabilidad de las aseguradoras (EPS). La nueva lista se definirá de forma negativa, es decir, enumerando servicios y medicamentos que definitivamente no deberán ser garantizados por la ley. Este es uno de los puntos más importantes de la Reforma en Salud, y su objetivo es que el grueso de los recobros pase ahora a ser administrado por las EPS de forma más competitiva, que la asignación de servicios no sea una decisión judicial y que el costo de los trámites administrativos sobre los pacientes y el Estado disminuya.

El objetivo de este artículo es cuantificar los costos y beneficios de este nuevo plan de salud para así hacer un análisis informado de los efectos de esta política. Por un lado, la unificación del POS y del No POS traerá consigo un aumento del gasto en salud. Esto se debe a que, al disminuir el costo de los trámites, muchos pacientes que no solicitaban medicamentos del No POS por los engorrosos trámites empezarán a solicitar servicios costosos a sus EPS. Por otro lado, el beneficio de los pacientes aumentará, pues quien lo requiera tendrá un acceso menos complicado a medicamentos especializados que antes era difícil adquirir. 
En este trabajo se usará un modelo de elección discreta para predecir las decisiones de los pacientes, respecto a solicitar o no servicios del No POS. Esto permitirá estimar el gasto en salud antes y después del nuevo diseño, y el excedente del consumidor antes y después del diseño. Con estas mediciones, se podrán calcular y comparar costos y beneficios del nuevo diseño. Debido a la forma en que se realiza el estudio, el cálculo de los costos y de los beneficios se podrá realizar sobre distintos grupos de la población, dividida por género, grupo de edad y diagnosis médica de enfermedades crónicas (de larga duración), lo que permitirá estudiar el efecto de la política sobre poblaciones en riesgo (algunos ejemplos son personas de la tercera edad, recién nacidos, enfermos renales crónicos, etc.).

Este estudio se centrará en el efecto sobre el Régimen Contributivo del Sistema de Salud. Esto se debe a que "el grueso de las tutelas y los servicios No POS se concentra en el régimen contributivo", como indican Guerrero y Bosset (2012, p. 34). Se simplificará el modelo, asumiendo que el precio de los servicios No POS no cambia después de su unificación con el POS. Este puede ser el efecto en el corto plazo, pero existe evidencia de que los servicios No POS han sido sobrefacturados sistemáticamente al no existir incentivos por parte de las EPS para contener sus costos. ${ }^{1}$ Así mismo, existe evidencia de que las imprecisiones del POS han permitido que las EPS nieguen servicios obligatorios para luego esperar el recobro del Estado. Con los datos es difícil detectar este fenómeno. La consecuencia de estas dos observaciones es que en el largo plazo los costos realizados pueden ser menores que los estimados, debido a que la competencia entre las EPS hará que el precio de los servicios que ahora son recobrados disminuya. Debido a esto, este estudio generará estimaciones del gasto consistentes en el corto plazo y que en el largo plazo son cotas superiores sobre el gasto total.

Este estudio tendrá en cuenta el problema que se genera al no poder observar en la base de datos cuál sería el gasto en servicios No POS generado por los pacientes que ahora únicamente usan servicios POS y que con la reforma entrarían a usar servicios del No POS. Además, se planteará el problema de forma probabilística, partiendo de que existe la probabilidad de que a un individuo se le nieguen servicios No POS solicitados.

Una limitación de este estudio, que deberá ser explorada con cortes longitudinales de los datos, es desconocer la elasticidad de sustitución entre servicios POS y servicios No POS. En un principio, se asume que estos son no sustitutos y las consecuencias son, de nuevo, que la estimación del gasto sea una cota superior sobre el cambio del gasto en el largo plazo y no una estimación exacta.

1 Esta observación se debe a Álvaro Muriel y Ramiro Guerrero. 


\section{Datos}

Se usa la información del gasto en servicios POS y servicios No POS durante 2010, de una muestra aleatoria de un millón de colombianos que hacen parte del régimen de salud contributivo. ${ }^{2}$ De cada individuo, se conoce su edad, género y el valor de 29 variables dummies que indican si el individuo sufre o no de alguna de las 29 enfermedades del grupo de enfermedades crónicas (de larga duración). Usando la edad, se divide la población en 12 grupos de edad, estos grupos son los que usa el gobierno para hacer el cálculo de la UPC y son construidos de forma tal que, dentro de un grupo de edad, los individuos tienen problemas de salud y gastos en salud similares. En el apéndice se puede encontrar la lista de 29 enfermedades crónicas.

El gasto en servicios POS reportado en la base de datos no es el adecuado para realizar las estimaciones. Esto se debe a que cerca del $50 \%$ de la muestra no cotizó durante todo el año, así que la variable no corresponde al gasto anual del individuo. Para corregir este problema, los datos fueron transformados haciendo una regla de tres que anualice el gasto. La regla fue aplicada para quien cotizó más de 30 días. Si el periodo de cotización fue menor a 30 días, se le dio el valor de 30 días a esta variable para evitar problemas de gastos anualizados inflados de forma exagerada.

En este estudio se hará referencia a grupos de riesgo de la población. A una enfermedad crónica, un grupo de edad y un género se le asocia un grupo de riesgo, que es el conjunto de individuos que sufren de esa enfermedad y pertenecen a ese grupo de edad y género. Así mismo, se crean grupos de riesgo para los individuos que no sufren ninguna enfermedad crónica y que pertenecen a un determinado grupo de edad y género. Esta definición tiene un problema y es que los grupos de riesgo no son disjuntos, al ser posible que un individuo sufra de dos o más enfermedades crónicas. Teniendo en cuenta que para la mayoría de la población este no es el caso, se simplifica el problema, asumiendo que cada paciente sufre únicamente de la enfermedad más costosa (donde el costo de una enfermedad se calcula como el promedio del gasto de los pacientes con esa enfermedad).

Es importante notar que con la base de datos solo se puede conocer cuánto gastaría una persona en servicios No POS, si ese individuo ya está accediendo a servicios No POS antes de la reforma. Esto significa que, en el caso de haber una reforma y que un individuo que no usa servicios del No POS pasara a consumirlos,

2 No se usa la base completa por los problemas computacionales que esto genera, pero la cantidad de datos usados garantiza una muy buena estimación de los costos. 
no se sabría cuánto gastaría. Esto es una clara limitación a un modelo de escogencia discreta, pues significa que no es posible calcular el beneficio que generan todas las elecciones de un agente. Debido a esto, una de las secciones se encarga de ajustar un modelo que estima el gasto potencial en servicios No POS para individuos que antes de la reforma únicamente usan el POS únicamente.

\section{Modelo de elección discreta}

Para modelar el problema de elección discreta se supone que el individuo $j$ debe escoger entre reclamar servicios del No POS o no reclamarlos. Concretamente, su decisión 1 consistirá en reclamar servicios del POS y en no reclamar servicios del No POS. Su decisión 2 consistirá en reclamar servicios del POS y servicios del No POS. ${ }^{3}$ Estas decisiones son mutuamente excluyentes y exhaustivas, como lo requiere un modelo de elección discreta.

Ahora, cada una de las decisiones reporta un beneficio en salud, pero también genera costos al individuo. El costo de la decisión 1 son todos los trámites y pagos que el paciente debe hacer para cotizar en el POS. Los costos de la decisión 2 incluyen los costos de la decisión 1 y, además, todos los costos asociados con la reclamación de servicios No POS. Se propone entonces el siguiente modelo para la utilidad generada por las dos decisiones.

$$
\begin{aligned}
& U_{1}=B_{1}-C_{P}+\varepsilon_{1} \\
& U_{2}=(1-p)\left(B_{2}-C_{P}-C_{N P}\right)+p\left(B_{1}-C_{P}-C_{N P}\right)+\varepsilon_{2}
\end{aligned}
$$

Donde $U_{i}$ denota la utilidad del individuo al tomar la decisión $i$ y $B_{i}$ denota el beneficio en salud del individuo asociado con tomar la decisión $i$. $C_{P}$ es el costo de cotizar en el POS y $C_{N P}$ es el costo de solicitar servicios No POS. El valor $p$ corresponde a la probabilidad de que los servicios No POS sean negados. Los valores $\varepsilon_{1}$ y $\varepsilon_{2}$ corresponden a los demás determinantes de la utilidad no observados.

Note que si el individuo toma la decisión 1 , recibirá un beneficio $B_{1}$ y deberá pagar los costos de acceso al POS. Si el individuo toma la decisión 2, recibirá un beneficio $B_{2}$ con una probabilidad $1-p$ y un beneficio $B_{1}$ con una probabilidad $p$. Este último escenario corresponde al momento en el que los servicios No POS son negados y el individuo deberá quedarse con el beneficio de haber decidido

3 No existen individuos que únicamente reclamen servicios No POS. Cuando un individuo reclama un servicio No POS, este siempre viene acompañado con la reclamación de un servicio POS. 
no reclamar. Es importante notar que, sin importar si el individuo recibe o no los servicios No POS al solicitarlos, este deberá asumir los costos $C_{N P}$ de reclamación, así como los costos $C_{p}$, pues la persona no deja de pagar el acceso al POS. Dado que todas las estimaciones se harán por grupo de riesgo y dentro de un grupo de riesgo las condiciones de salud son homogéneas, se asumirá que $p$ es constante entre individuos.

Si un servicio es negado, el individuo tiene dos opciones, costear el servicio con su dinero o no usarlo. Debido a que los datos no permiten determinar cuál fue la decisión que tomó el individuo si su servicio fue negado, estas opciones no son tomadas en cuenta. Esto no tiene serios efectos sobre las estimaciones en el corto plazo, pues cualquiera que sea la decisión que tome el individuo, no se afecta el gasto del Estado. En el largo plazo puede que sí, pues si al individuo se le niega un servicio importante, puede que se aumente el gasto futuro por complicaciones o porque el servicio es sustituido por servicios del POS.

Ahora, al calcular la diferencia entre las utilidades se obtiene:

$$
U_{2}-U_{1}=(1-p)\left(B_{2}-B_{1}\right)-C_{N P}+\left(\varepsilon_{2}-\varepsilon_{1}\right)
$$

Se espera que los beneficios en salud $B_{2}$ y $B_{1}$ que recibe el individuo dependan positivamente del gasto que reportaría este individuo en el sistema. Esto se explica en que los procedimientos y servicios de mayor calidad y variedad generan un mayor beneficio en salud, pero, al mismo tiempo, un mayor costo en el sistema. Esta dependencia será modelada por conveniencia linealmente, de modo que se supondrá que:

$$
\begin{aligned}
& B_{1}=\beta_{G R} \text { Gasto }_{1} \\
& B_{2}=\beta_{G R} \text { Gasto }_{2}
\end{aligned}
$$

Donde Gasto ${ }_{1}$ indica el gasto en salud si el individuo no recibe servicios No POS y Gasto $_{2}$ indica el gasto si los recibe. Aquí la constante $\beta_{G R}$ depende del grupo de riesgo que se esté estimando. Reemplazando estos valores, se obtiene:

$$
U_{2}-U_{1}=\beta_{G R}(1-p)\left(\text { Gasto }_{2}-\text { Gasto }_{1}\right)-C_{N P}+\left(\varepsilon_{2}-\varepsilon_{1}\right)
$$

La probabilidad de que un individuo tome la decisión 2 es,

$$
P\left(U_{2}-U_{1}>0\right)=P\left(\varepsilon 1-\varepsilon 2<\beta_{G R}(1-p)\left(\text { Gasto }_{2}-\text { Gasto }_{1}\right)-C_{N P}\right)
$$

Asumiendo que $F$ es la distribución de $\varepsilon_{1}-\varepsilon_{2}$ y definiendo $\gamma=\beta_{G R}(1-p)$ 


$$
P\left(U_{2}-U_{1}>0\right)=F\left(\gamma\left(\text { Gasto }_{2}-\text { Gasto }_{1}\right)-C_{N P}\right)
$$

Esta ecuación tiene una explicación muy intuitiva. La diferencia $\mathrm{Gasto}_{2}-$ Gasto $_{1}$ indica cuánto más puede gastar un paciente reclamando servicios No POS que no reclamándolos. Como mayor gasto está relacionado con mayor calidad (dejando los niveles de eficiencia constantes), una mayor diferencia de este gasto atrae más al paciente a tomar la decisión 2. Por otro lado, un aumento en $C_{N P}$ significa mayores costos de acceder a servicios No POS, luego, menores incentivos para tomar la decisión 2.

La ecuación (1) será estimada por máxima verosimilitud. Para esto, se asumirá que los errores son GEV y que por lo tanto $F$ es una función logística.

\section{Ajuste del modelo}

El ajuste del modelo a la base se realiza en dos etapas. La primera etapa consiste en la estimación de los gastos para individuos que toman la decisión 2, la segunda etapa consiste en la división de la base en grupos de riesgo y en la estimación de los parámetros de la ecuación (1) por máxima verosimilitud.

\subsection{Estimación del Gasto}

Para los individuos que toman la decisión 2 y logran cobrar servicios No POS se conoce el gasto total y se puede calcular el gasto de haber tomado la decisión 1 como el gasto agregado en servicios POS. Un supuesto importante de este cálculo es que, de tomar la decisión 1 , los individuos gastarán lo mismo que gastan ahora en servicios POS. Esto puede no mantenerse, pues de no tener acceso a servicios No POS podría haber una sustitución por servicios del POS, lo que haría que el gasto en POS fuera mayor.

Para los individuos que toman la decisión 1 se desconoce el gasto en salud al tomar la decisión 2. Este gasto se necesita para ajustar un modelo de elección discreta, así que se estimará asumiendo el siguiente modelo

$$
G_{2}=\alpha_{G R} G_{1}+\eta
$$

donde se asume que la constante $\alpha_{G R}$ depende del grupo de riesgo.

Es importante discutir los supuestos de este modelo. Primero, se asume que el gasto de la decisión 1 y la decisión 2 son proporcionales y están relacionados linealmente. La razón es que ambas cantidades están fuertemente correlacionadas con el estado de salud del individuo. Un individuo sano reportará bajos 
gastos, ya sea que use o no servicios del No POS. De la misma forma, un individuo muy enfermo reportará altos costos, ya sea que use o no servicios del POS. Esto implica que los dos gastos están correlacionados y su relación se puede aproximar de forma lineal.

Segundo, se asume que el factor de proporcionalidad $\alpha_{G R}$ es constante dentro del grupo de riesgo. Esto significa que después de controlar por género, edad y diagnosis, la relación entre los gastos es similar. Este supuesto parte de que dentro de un grupo de riesgo, los individuos presentan condiciones de salud similares y se puede asumir que la distribución de los gastos es la misma.

La estimación de este modelo se hace por $\mathrm{MCO}$, al usar una constante $\alpha_{G R}$ distinta para cada grupo de riesgo. La definición de grupo de riesgo puede encontrarse en la sección de Datos.

\subsection{Estimación de los parámetros del modelo}

Se debe estimar la siguiente ecuación por máxima verosimilitud

$$
P\left(U_{2}-U_{1}>0\right)=F\left(\gamma_{G R}\left(\text { Gasto }_{2}-\text { Gasto }_{1}\right)-C_{N P}\right)
$$

Los parámetros $\gamma_{G R}$ y $C_{N P}$ deberán variar entre grupos de riesgo, pues el impacto del gasto sobre la utilidad y los costos de reclamar servicios No POS varían dependiendo de la diagnosis, el grupo de edad y el género al que pertenece cada paciente.

Debido a esto, se divide la población en grupos de riesgo y dentro de cada grupo de riesgo se estiman los parámetros correspondientes por máxima verosimilitud asumiendo que la función de distribución $F$ es un logit.

Puede ocurrir que existan variables no observadas que afecten la decisión de un individuo. Por ejemplo, si un individuo no tiene educación puede que desconozca los mecanismos para realizar una tutela. Sin embargo, al momento de estimar los parámetros, basta que estas variables no estén correlacionadas con el nivel de gasto. Para poder hacer la estimación, se asume que dentro de un grupo de riesgo no existe una fuerte correlación entre el nivel de gasto y las variables no observadas (por ejemplo, si se asume que el nivel de gravedad de la enfermedad no está fuertemente relacionada con el nivel de educación). Esto es sensato, dado que después de controlar por enfermedad, género y edad, el gasto debería comportarse de forma casi aleatoria.

Se escogió la estimación de los parámetros por grupo de riesgo frente a otras alternativas, como la estimación de un logit a toda la base, por varias razones. Primero, los costos de acceder a servicios No POS $C_{N P}$ deben variar entre grupos de riesgo, pues es más fácil para un anciano asmático, por ejemplo, acceder 
a medicamentos especializados mediante una tutela, que para un joven sin enfermedades crónicas. Segundo, se evita el problema de heteroscedasticidad asociado con estimar un logit a toda la base, pues la varianza de los errores es mayor para pacientes crónicos, con mayor variabilidad de enfermedades y de gasto, que para pacientes no crónicos, por ejemplo. Tercero, el gran tamaño de la base permite una estimación buena de los parámetros y no es necesario usar modelos basados en distribuciones GEV o logits mixtos para corregir el problema de la falta de datos.

Tras la estimación, los signos fueron los esperados y todas las variables de control fueron significativas al 5\% en cerca del $80 \%$ de los grupos de riesgo.

\section{Evaluación de la política}

La unificación de los servicios del POS y del No POS en un único plan facilitará los trámites y disminuirá los costos de acceso a servicios que no están ahora cubiertos por el Plan Obligatorio de Salud. Desde el punto de vista de la ecuación (1), la política implica una reducción en $C_{N P} \mathrm{y}$, por lo tanto, un cambio en la probabilidad de escoger la decisión 2.

Si los costos de acceder a servicios No POS se reducen a una fracción $c$ de lo que eran originalmente, la probabilidad de escoger la decisión 2 estará dada por:

$$
P_{c}\left(U_{2}>U_{1}\right)=F\left(\gamma_{G R}\left(\text { Gasto }_{2}-\text { Gasto }_{1}\right)-c C_{N P}\right)
$$

Donde $P_{c}$ indica la probabilidad de escoger la decisión 2 cuando los costos del No POS se han reducido a una fracción $c$ de lo que eran originalmente. A continuación, se describirá cómo a partir de la ecuación (2) es posible estimar la variación del gasto en salud y del excedente del consumidor de toda la población después de la reforma.

\subsection{Gasto}

El gasto esperado del individuo $i$ está dado por

$$
E\left[\text { Gasto }_{i}\right]=p_{i} \text { Gasto }_{i 2}+\left(1-p_{i}\right) \text { Gasto }_{i 1}
$$

Y el gasto esperado después de una reducción de los costos de acceso al No POS será

$$
E c\left[\text { Gasto }_{i}\right]=p_{c i} \text { Gasto }_{i 2}+\left(1-p_{c i}\right) \text { Gasto }_{i 1}
$$


así que los gastos totales antes y después de la reforma serán

$$
\begin{aligned}
& E[\text { GastoTotal }]=\sum_{i=1}^{n} E\left[\text { Gasto }_{i}\right] \\
& E_{c}[\text { GastoTotal }]=\sum_{i=1}^{n} E_{c}\left[\text { Gasto }_{i}\right]
\end{aligned}
$$

Con estas ecuaciones se obtiene el gasto esperado antes y después de la reforma y se calcula la variación porcentual en el gasto total.

\subsection{Bienestar}

En general, el análisis del cambio en bienestar generado por una política es complicado. A partir de un modelo de elección discreta es posible calcular cambios en niveles de utilidad, pero estos cambios son, en principio, no comparables. Bajo ciertos supuestos, sin embargo, es posible hacer un cálculo del cambio en el excedente del consumidor generado por una política. Estos supuestos se ajustan perfectamente a este modelo. La metodología seguirá a Train (2009).

El individuo $i$ se enfrenta a dos decisiones que le representan una utilidad $U_{i 1}=V_{i 1}+\varepsilon_{i 1}$ y $U_{i 2}=V_{i 2}+\varepsilon_{i 2}$. Aquí $V_{i j}$ denota la utilidad explicada por el gasto en salud de la decisión $j$, también llamada parte observable de la utilidad. La utilidad del individuo será la de la decisión que le genere un mayor beneficio, es decir, $U_{i}=\max \left(U_{i 1}, U_{i 2}\right)$. Bajo el supuesto de errores GEV i.i.d de Small y Rosen (1981) se muestra que el valor esperado del beneficio será:

$$
E\left[U_{i}\right]=E\left[\max \left(U_{i 1}, U_{i 2}\right)\right]=\ln \left(\exp \left(\mathrm{V}_{\mathrm{i} 1}\right)+\exp \left(\mathrm{V}_{\mathrm{i} 2}\right)\right)
$$

Si se usa $A$ para indicar valores antes y $P$ para valores después de una reforma se tendrá que el cambio en utilidad generado por la reforma será:

$$
E\left[\quad U_{i}\right]=E\left[U_{i}^{P}\right]-E\left[U_{i}^{A}\right]=\ln \left(\exp \left(V_{i 1}^{P}\right)+\exp \left(V_{i 2}^{P}\right)\right)-\ln \left(\exp \left(V_{i 1}^{A}\right)+\exp \left(V_{i 2}^{A}\right)\right)
$$

Finalmente, si se busca comparar niveles de utilidad entre individuos es necesario transformarlos a unidades monetarias. Si $m_{i}$ es la utilidad marginal del ingreso, el cambio en utilidad transformado a unidades monetarias estará dado por: 


$$
E\left[C S_{i}\right]=\frac{E\left[\Delta U_{i}\right]}{m_{i}}
$$

Este valor será el excedente del consumidor y será denotado por CS, por sus siglas en inglés. Para reemplazar $m_{i}$ se usa el $\gamma_{G R}$ estimado en la ecuación (1), pues esta cantidad corresponde a la utilidad marginal del gasto sobre la utilidad.

\section{Resultados}

Se realiza el cálculo del cambio en el gasto y en el excedente del consumidor cuando la reforma disminuye los costos de acceder a servicios No POS en distintas proporciones. Los resultados se muestran en la tabla 1. Las desviaciones corresponden a la varianza entre grupos de riesgo.

La ventaja de la metodología usada es que permite calcular los cambios en el gasto y en el excedente por cada grupo de la población. Es posible entonces identificar qué grupos se beneficiarán más con la política y qué grupos reportarán un mayor aumento en el gasto. Las tablas 3 y 4 presentan grupos que reportan alto, medio y bajo incremento en estas categorías.

Los grupos para los que se encuentra un mayor aumento proporcional en el excedente del consumidor con relación al gasto fueron los individuos sin enfermedades crónicas. Esto se debe a por lo menos dos razones: primero, los individuos que tienen enfermedades crónicas tienen una alta probabilidad de reclamar servicios del No POS antes de la reforma, por lo que después de la reforma la cantidad de individuos que cambian sus preferencias, es decir, que pasan a reclamar servicios del No POS es baja comparada con la de individuos sin enfermedades crónicas. Segundo, para un individuo que sufre de enfermedades crónicas, los costos de acceder al No POS son más pequeños que para un individuo que no sufre de enfermedades crónicas (una tutela, por ejemplo, tiene mayor probabilidad de ser aprobada para el primer individuo que para el segundo). Esto hace que una reducción en los costos del No POS tenga un mayor impacto sobre el individuo no crónico que sobre el crónico.

Es importante tener en cuenta que los individuos que no tienen enfermedades crónicas reportan mayores cambios proporcionales en el gasto, pero esto no implica mayores cambios en el gasto absoluto. De hecho, el grueso del gasto está y permanecerá en los individuos que sufren de enfermedades crónicas.

Una cantidad interesante es la relación entre el cambio en el excedente y el cambio en el gasto. Este factor muestra cómo el gasto neto en salud se multiplica para convertirse en excedente. Es una muy buena medida de la eficiencia de la 
política. La tabla 2 muestra valores de esta relación para toda la población y la tabla 5 muestra valores para unos cuantos grupos.

\section{Conclusiones}

El aumento del gasto en salud tras la reforma dependerá de cuánto se relajen los costos de acceder a servicios No POS tras el cambio en el diseño del sistema. Como lo muestra la tabla 1, este aumento es como máximo del 16\%, lo cual representa de por sí una fracción alta, sin embargo, relajaciones menos fuertes, como la del 50\%, llevarán a un aumento del gasto en salud de cerca del $4,7 \%$.

El aumento del excedente del consumidor es mucho más alto que el aumento del costo. La tabla 2 indica que para algunas relajaciones la relación entre el aumento del excedente y el aumento del gasto puede llegar a ser de 7 a 1 . Estos valores sugieren que el nuevo plan de beneficios tiene ganancias muy altas para la población.

Es posible hacer un análisis global de la política y un análisis local, concentrándose en cada uno de los grupos de riesgo. El análisis global, que compara cambios absolutos en gasto y en excedente, muestra que la política es altamente eficiente. Analizando cada uno de los grupos de riesgo, es fácil notar que existen poblaciones que se benefician más que otras. Por ejemplo, los enfermos no crónicos tendrán un aumento en su excedente mucho mayor que el de los enfermos crónicos. Entonces, aparece una pregunta normativa ¿el Gobierno estará dispuesto a costear el aumento del gasto de una población no vulnerable como lo son los enfermos no crónicos para procurarles un aumento considerable en su bienestar?

La herramienta desarrollada en este estudio permite hacer un análisis detallado del efecto del nuevo diseño del sistema de salud para cada uno de los grupos de riesgo en los que se divide la población, sin embargo, presenta ciertas debilidades en las que se debe trabajar. Las más importantes son, primero, lograr modelar la elasticidad de sustitución entre servicios POS y No POS, segundo, determinar los efectos que tendrá la unificación del POS y el No POS sobre los precios de los servicios que ahora son recobrados al Gobierno y que solo se obtienen por medio de tutelas. Estos dos problemas han tenido como consecuencia que las estimaciones sean válidas en el corto plazo y que en el largo plazo sean cotas superiores sobre el aumento del gasto del Gobierno. 


\section{Referencias}

Guerrero, R. \& T. Bossert. (2012). Diseño y reforma del Plan Obligatorio de Salud en Colombia. Documentos PROESA 2, 1-45

McFadden, D. (1974). Conditional logit analysis of qualitative choice behavior. In P. Zarembka, (Ed.), Frontiers in Econometrics (pp. 105-142). New York: Academic Press.

Small, K., \& Rosen, H. (1981). Applied welfare economics of discrete choice models. Econometrica, 49, 105-130.

Train, K. (2009). Discrete Choice Methods with Simulation. Cambridge: Cambridge University Press.

Williams, H. (1977). On the formation of travel demand models and economic evaluation measures of user benefits. Environment and Planning, A 9, 285-344.

\section{Anexo}

\section{Enfermedades Crónicas}

Los siguientes fueron los grupos de enfermedades construidos a partir de la clasificación CIE10:

Anomalías genéticas y congénitas, artritis, artritis piógenas y reactivas, artrosis, asma, autoinmune, cáncer cérvix in-situ, cáncer cérvix invasivo, cáncer genitales masculinos, cáncer mama, cáncer melanoma y de la piel, cáncer órganos digestivos, cáncer órganos respiratorios, cáncer otros cáncer, cáncer otros genitales femeninos, cáncer tejido linfático y afines, cáncer terapia cáncer, diabetes, enfermedad cardiovascular - hipertensión, enfermedad cardiovascular - otra, enfermedad pulmonar de larga duración, renal - insuficiencia renal crónica, renal - insuficiencia renal otra, renal - otra, renal - renal de larga duración, sida-VIH, síndromes convulsivos (epilepsia), trasplante, tuberculosis.

Tabla1. Cambios en el gasto y en el excedente para distintos valores de c

\begin{tabular}{rrrcc}
\hline Factor c & A. Gasto & Desviación & A. Excedente & Desviación \\
\hline $0 \%$ & $16,05 \%$ & $7,10 \%$ & $91,94 \%$ & $120,21 \%$ \\
$25 \%$ & $7,36 \%$ & $4,06 \%$ & $53,17 \%$ & $59,06 \%$ \\
$50 \%$ & $4,68 \%$ & $2,52 \%$ & $31,16 \%$ & $31,91 \%$ \\
$75 \%$ & $2,40 \%$ & $1,17 \%$ & $13,78 \%$ & $13,41 \%$ \\
\hline
\end{tabular}


Tabla 2. Relación entre aumento del gasto y del excedente para distintos valores de c

\begin{tabular}{cc}
\hline Factor c & Relación \\
\hline $0 \%$ & 5,73 \\
$25 \%$ & 7,22 \\
$50 \%$ & 6,65 \\
$75 \%$ & 5,74 \\
\hline
\end{tabular}

Tabla 3. Aumentos en el gasto para distintos grupos

\begin{tabular}{ccccc}
\hline Edad & Sexo & Enfermedad & A. Gasto c $=25 \%$ & A. Gasto c $=50 \%$ \\
\hline $70-74$ & M & No crónico & $14,75 \%$ & $9,36 \%$ \\
$19-44$ & M & Artritis & $6,85 \%$ & $4,49 \%$ \\
$60-64$ & F & Diabetes & $3,79 \%$ & $2,53 \%$ \\
\hline
\end{tabular}

Tabla 4. Aumentos en el excedente para distintos grupos

\begin{tabular}{ccccc}
\hline Edad & Sexo & Enfermedad & A. Excedente c $=25 \%$ & A. Excedente c $=50 \%$ \\
\hline $5-14$ & M & No crónico & $293 \%$ & $142 \%$ \\
$5-14$ & F & Epilepsia & $41 \%$ & $25 \%$ \\
$15-18$ & F & Cáncer mama & $2,9 \%$ & $1,6 \%$ \\
\hline
\end{tabular}

Tabla 5. Relación entre aumento del gasto y del excedente para distintos grupos

\begin{tabular}{ccccc}
\hline Edad & Sexo & Enfermedad & Relación c $=25 \%$ & Relación c $=50 \%$ \\
\hline $65-69$ & M & Trasplante & 33,79 & 59,3 \\
$65-69$ & M & Artritis & 10,17 & 9,54 \\
$55-59$ & F & Tuberculosis & 2,48 & 2,01 \\
\hline
\end{tabular}

